\title{
An end to antimicrobial prophylaxis against infective endocarditis for dental procedures?
}

\author{
M. V. Martin
}

In 2005/6 a working party of the British Society for Antimicrobial Chemotherapy (BSAC), after considering the evidence for the use of antimicrobial prophylaxis for dental patients at risk of infective endocarditis, felt that it was not justified. ${ }^{1}$ They considered that the total abolition of the current guidelines was too drastic and therefore only certain groups of patients were recommended to receive antimicrobial prophylaxis. This advice did not find favour with a number of medical professionals including some, but not all, cardiologists. In response to the controversy, consideration of the available evidence was referred to the National Institute for Clinical Excellence (NICE), who published their conclusions, not just for dentistry, but for other surgical interventions in November 2007 for consultation. ${ }^{2}$

NICE considered the possibility of defining risk groups of patents who are more likely to develop IE and identified four, those with: acquired valvular disease (including stenosis and regurgitation), valve replacement, structural congenital heart disease and hypertrophic cardiomyopathy. The evidence for these conclusions is clearly presented and documented, providing a major help to practising clinicians in defining risk.

\section{THE ROLE OF BACTERAEMIAS}

The role of bacteraemias in the pathogenesis of IE was also considered. In particular, the evidence as to whether IE is caused by bacteraemias after dental procedures, or other normal activities such as tooth brushing. The seminal work of Roberts played a large part in the conclusion by NICE that IE was unlikely to be caused by any single dental procedure. ${ }^{3}$ IE could occur at any time in individuals at risk without there being a single responsible surgical event. NICE recognised the essential and pivotal roles of the individual and the dental professional in maintaining the oral health of patients at risk of IE. This is the second scientific enquiry (the first was the BSAC in 2006) that recognised that the link between dentistry and IE is at best unproven and unsupported by science. The conclusion from this has to be that it is impossible to precisely define a single dental procedure, whether it causes a bacteraemia or not, that is likely to cause IE.

NICE reported that there was no evidence to support the contention that antibiotic prophylaxis reduces bacteraemias, although it may reduce the frequency of their detection after the procedure; concluding that it was therefore not possible to determine its effect on bacteraemias. There is further consideration of the effect of chlorhexidine rinses on bacteraemias in patients at risk of IE. Again, NICE could not decide whether or not chlorhexidine reduces the incidence of post-operative bacteraemias. On the basis of this and other evidence NICE makes a clear and unequivocal recommendation in the consultation document that neither antibiotic prophylaxis, nor chlorhexidine should be given before any dental procedures in patients at risk of IE.

Having compiled all the available data on economic factors the group concludes that if amoxicillin prophylaxis was effective the cost of preventing one case of IE would be approximately $£ 12$ million. It was found impossible to calculate the cost of preventing one death from IE as no data could be found to support the contention that amoxicillin would be effective. The adverse affects of amoxicillin (ie anaphylaxis) are also discussed and found to far outweigh any benefits.

It is to be hoped that the consultation will have stimulated considered and scientifically-supported responses so the debate can move constructively forward. This is no time for emotion and unsupported contentions to cloud what is a substantial and unbiased consideration of the available science by a respected, unbiased group. NICE will publish their definitive recommendations next month and if there are no substantive scientific objections then effectively March 2008 will see an end to antimicrobial prophylaxis for patients who were considered at risk of IE after dental procedures. Perhaps it will also bring to an end both the threat of, and actual, litigation involving dentistry and IE. ${ }^{4}$ It will however pose some difficulties in communication for dentists who have previously recommended antimicrobial prophylaxis, as patients may need convincing that that there is no longer a need. Instead, perhaps NICE will focus the emphasis for dentists on where it should be, making sure patients with cardiac conditions have and maintain good oral health.

\section{m.v.martin@btinternet.com}

1. Gould F K, Elliott T S, Fowreaker J et al. Guidelines for the prevention of endocarditis: report of the Working Party of the British Society for Antimicrobial Chemotherapy. J Antimicrob Chemother 2006: 57: 1035-1042.

2 NICE guidelines on prophylaxis against infective endocarditis http://www.nice. org.uk/nicemedia/pdf/PIEGuidelines.pdf

3. Roberts G J. Dentists are innocent! "Everyday" bacteraemia is the real culprit: a review and assessment of the evidence that dental surgical procedures are the principal cause of infective endocarditis in children. Paediatr Cardiol 1999; 20: 317-325.

4. Martin M V, Longman L P, Forde M P, Butterworth M L. Infective endocarditis and dentistry: the legal basis for an association. Br Dent J 2007; 203: E1 